\title{
TEM Characterization of Epitaxial MnSi Films Grown on (111) Si Substrates
}

\author{
M. D. Robertson,* E. A. Karhu** and T. L. Monchesky** \\ * Dept. of Physics, Acadia University, Wolfville, NS B4P 2R6, Canada \\ ** Dept. of Physics and Atmospheric Science, Dalhousie University, Halifax, NS B3H 3J5, Canada
}

Manganese silicide (MnSi) has recently attracted significant attention as an ideal system for studying spin-dependent transport in helical magnets. The unit cell of $\mathrm{MnSi}$ is non-centrosymmetric with space group $\mathrm{P} 2{ }_{1} 3$ and a lattice parameter of $0.4558 \mathrm{~nm}$ [1]. In bulk form and at ambient pressure, the material forms a helical magnet at a Curie temperature of $29.5 \mathrm{~K}$. However, in the presence of tensile strain arising from epitaxy with a (111) silicon substrate, Curie temperatures $50 \%$ larger than the bulk have been observed. In order to better understand the structure-magnetic property relationship in greater detail, transmission electron microscopy (TEM) has been used to characterize 4-21 nm thick epitaxial layers of MnSi grown on Si (111) substrates by solid-phase epitaxy. The tensile strain in the layers is a result of the $3.0 \%$ lattice mismatch between MnSi (111) and Si (111) when the MnSi (111) layer is rotated $30^{\circ}$ with respect to the substrate so that the $[\overline{1} 2 \overline{1}]$ direction in $\mathrm{MnSi}$ is parallel to [10 $\overline{1}]$ of $\mathrm{Si}$.

Fig. 1(a) is a plan-view selected-area-electron-diffraction pattern (SADP) of an MnSi layer on Si (111) where this epitaxial relationship has been observed. The innermost circle of diffraction spots correspond to MnSi $\{101\}$ reflections and the second circle of spots to overlapping MnSi $\{112\}$ and $\mathrm{Si}\{220\}$ reflections. Fig. 1(b) is an enlarged view of one of the MnSi $\{101\}$ reflections where the central spot is due to MnSi $\{101\}$ and the hexagonal outer circle of diffraction spots are due to double diffraction effects with the Si $\{220\}$ reflections. The spacing between the central spot and the outer ring of spots can be used to accurately determine the level of strain in the MnSi layers. Using the Si reflections as an internal calibration standard, the residual in-plane strain in the MnSi films was measured as a function of film thickness and is presented in Fig. 1(c). The residual tensile strain in the film decreased monotonically with film thickness and the remainder of the interfacial lattice misfit is believed to be accommodated by misfit dislocations at the interface.

Since the unit cell of MnSi does not possess a centre of symmetry, the helical magnet can be oriented with either right- or left-handed chirality as a result of the ordering of $\mathrm{Mn}$ atoms along the [111] direction. Presented in Fig. 2 is a series of bright and dark-field images of an $18 \mathrm{~nm}$ thick MnSi layer which highlights strong diffraction contrast that can arise between inversion domains. Fig. 2(a) is a bright-field image and no inversion domain contrast is observed as expected from theory. The diffraction conditions used to obtain these images is shown in Fig. 2(b). The sample was tilted about 40 degrees off of the [111] zone-axis orientation in order to increase the effective layer thickness with respect to the electron beam and maximize image contrast. Furthermore, the sample was tilted so that no strong reflections from the Si substrate were present. Displayed in Fig. 2(c) and (d) are dark-field images obtained using reflections with a complex structure factor that display strong diffraction contrast as a result of domains with unit cells that are related to one another by an inversion transformation. Image and diffraction simulations are presently underway to determine the chirality of individual domains. 
References

[1] T. Jeong and W. E. Pickett, Phys. Rev. B, 70 (2004) 075114.

[2] This work was financially supported by the Natural Sciences and Engineering Research Council of Canada and the Canada Research Chairs program.

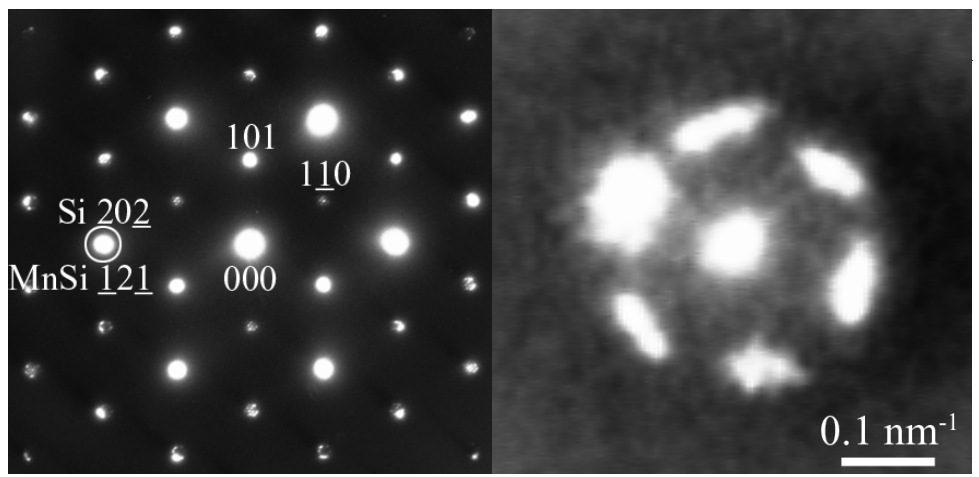

(a) (b)

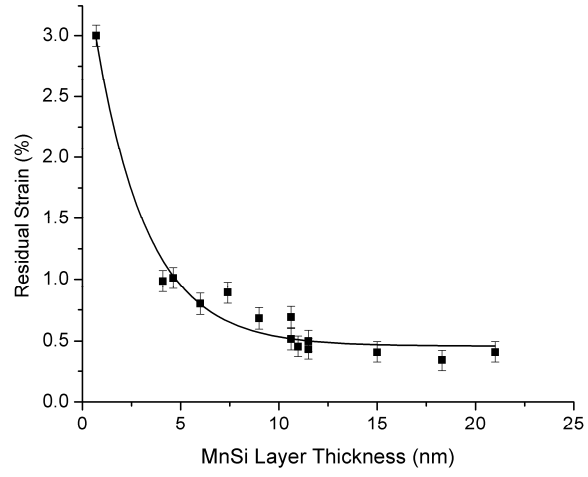

(c)

FIG. 1. (a) SADP of an epitaxial MnSi layer on Si (111). (b) An enlarged view of one of the MnSi $\{101\}$ reflections. (c) A plot of the in-plane residual strain as a function of layer thickness.

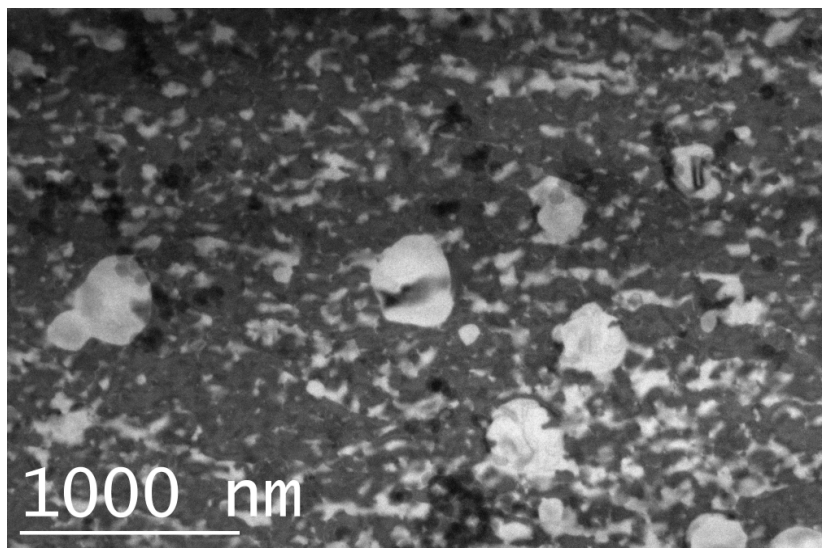

(a)

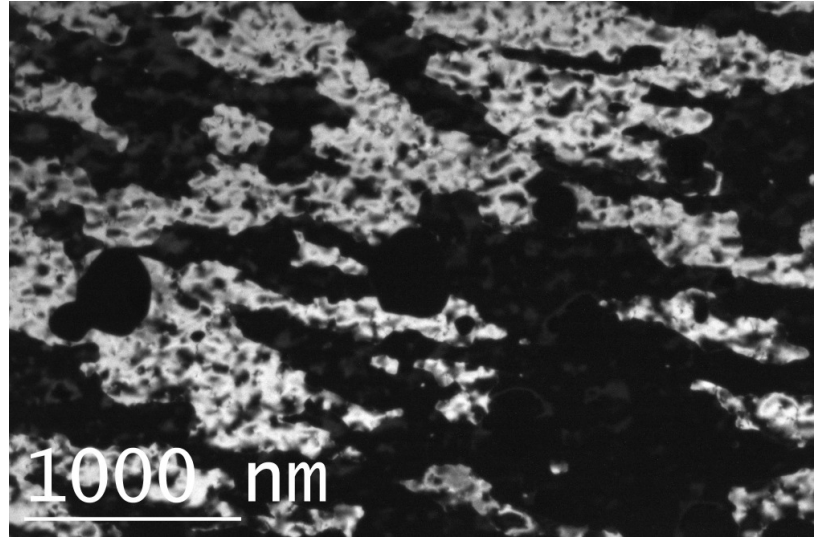

(c)

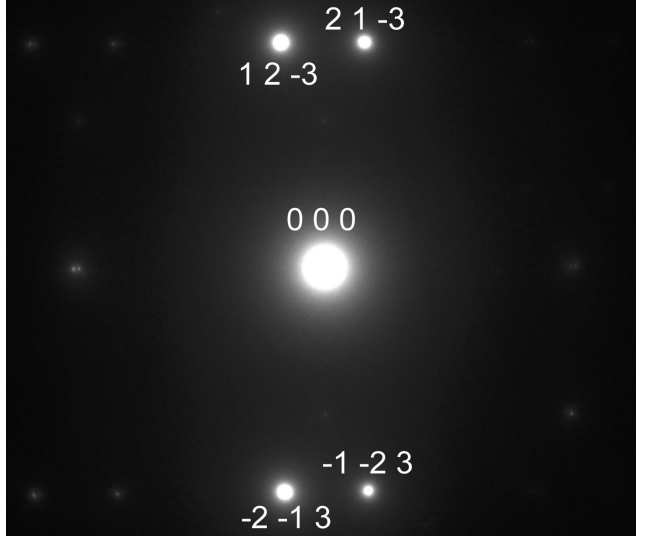

(b)

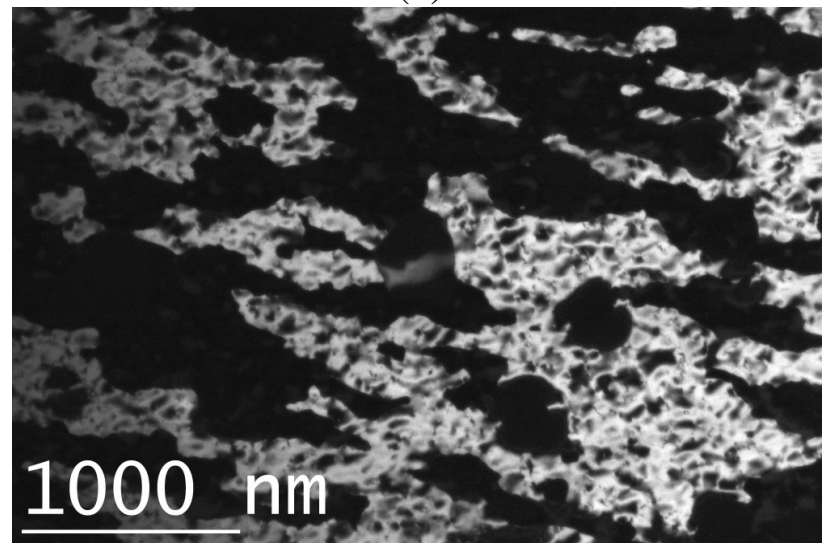

(d)

FIG. 2. (a) Plan-view bright-field image of an $18 \mathrm{~nm}$ thick $\mathrm{MnSi}$ layer using the diffraction conditions in (b). (c) and (d) Dark-field images using the $12-3$ and 21 -3 reflections, respectively. 